\title{
Selective ablation of basophils in mice reveals their nonredundant role in acquired immunity against ticks
}

\author{
Takeshi Wada, ${ }^{1}$ Kenji Ishiwata, ${ }^{2}$ Haruhiko Koseki, ${ }^{3}$ Tomoyuki Ishikura, ${ }^{3}$ Tsukasa Ugajin,, \\ Naotsugu Ohnuma, ${ }^{1}$ Kazushige Obata, ${ }^{1}$ Ryosuke Ishikawa, ${ }^{1}$ Soichiro Yoshikawa, ${ }^{1}$ \\ Kaori Mukai, ${ }^{1}$ Yohei Kawano, ${ }^{1}$ Yoshiyuki Minegishi, ${ }^{1,5}$ Hiroo Yokozeki, ${ }^{4}$ \\ Naohiro Watanabe, ${ }^{2}$ and Hajime Karasuyama ${ }^{1,5}$
}

\begin{abstract}
${ }^{1}$ Department of Immune Regulation, Tokyo Medical and Dental University Graduate School, Tokyo, Japan. ${ }^{2}$ Department of Tropical Medicine, Jikei University School of Medicine, Tokyo, Japan. ${ }^{3}$ Laboratory for Developmental Genetics, RIKEN Research Center for Allergy and Immunology, Yokohama, Japan. ${ }^{4}$ Department of Dermatology and ${ }^{5}$ Japan Science and Technology Agency (JST), Core Research for Evolutional Science and Technology (CREST), Tokyo Medical and Dental University Graduate School, Tokyo, Japan.
\end{abstract}

\begin{abstract}
Ticks are ectoparasitic arthropods that can transmit a variety of microorganisms to humans and animals during blood feeding, causing serious infectious disorders, including Lyme disease. Acaricides are pharmacologic agents that kill ticks. The emergence of acaricide-resistant ticks calls for alternative control strategies for ticks and tick-borne diseases. Many animals develop resistance to ticks after repeated infestations, but the nature of this acquired anti-tick immunity remains poorly understood. Here we investigated the cellular and molecular mechanisms underlying acquired resistance to Haemaphysalis longicornis ticks in mice and found that antibodies were required, as was IgFc receptor expression on basophils but not on mast cells. The infiltration of basophils at tick-feeding sites occurred during the second, but not the first, tick infestation. To assess the requirement for basophil infiltration to acquired tick resistance, mice expressing the human diphtheria toxin receptor under the control of the mast cell protease $8(\mathrm{Mcpt8})$ promoter were generated. Diphtheria toxin administration to these mice selectively ablated basophils. Diphtheria toxin-mediated basophil depletion before the second tick infestation resulted in loss of acquired tick resistance. These data provide the first clear evidence, to our knowledge, that basophils play an essential and nonredundant role in antibody-mediated acquired immunity against ticks, which may suggest new strategies for controlling tick-borne diseases.
\end{abstract}

\section{Introduction}

Ticks, particularly members of the Ixodid family, are blood-feeding ectoparasites of vertebrates and are also important vectors of pathogens, including virus, bacteria, protozoa, and helminths, which can cause several serious infectious diseases in humans and animals $(1,2)$. Ticks suck a blood meal from hosts over a period of several days and increase their body weight up to 120 -fold. Once they replete with blood meal (engorgement), ticks drop off from the host. While feeding, various substances of ticks are injected into the host to facilitate successful blood feeding, including a cement to anchor the mouth parts to the skin of the host, enzymes, vasodilators, and antiinflammatory, antihemostatic, and immunosuppressive substances (3-5). Pathogens can be transmitted from infected ticks to the host during salivation. Tick-transmitted diseases include viral encephalitis, Lyme disease caused by spirochetes of the Borrelia burgdorferi, Rocky Mountain spotted fever caused by Rickettsia rickettsii, monocytic human ehrlichiosis caused by Ebrlichia chaffeensis, and babesiosis caused by protozoa Babesia (1). The public health importance of ticks is not diminishing, and the emergence of acaricide-resistant ticks has shifted the anti-tick strategy toward the immunological control of ticks (6-8).

Several species of animals, including guinea pigs, rabbits, bovines, and mice, have been shown to develop resistance to tick feeding after a single or multiple infestation with ticks (6), which

Conflict of interest: The authors have declared that no conflict of interest exists. Citation for this article: J Clin Invest. 2010;120(8):2867-2875. doi:10.1172/JCI42680. is characterized by reduced numbers and weights of engorged ticks or tick death in subsequent infestations. Acquired host resistance to tick infestation was first documented by Trager in 1938 (9) and extensively studied by using guinea pigs in the 1970s and 1980s. It can be transferred to naive animals with cells or sera isolated from infested animals, and therefore the resistance is considered to be a type of immunological reaction (9-11). Immune resistance to the ticks is an important element of protection from infestation with these ectoparasites and also contributes significantly to the reduction in pathogen transmission from infected ticks (12-15). This is the rationale for the development of tick antigen-based vaccines to prevent tick-borne diseases. However, the nature of naturally acquired protective immunity against ticks remains ill defined. The tick-feeding sites in guinea pigs that had already experienced tick infestation were characterized by large accumulation of basophils and eosinophils, and basophils compose up to $70 \%$ of the infiltrating cells $(10,11,16)$. One study reported that the treatment of such guinea pigs with basophil-depleting antiserum abolished tick resistance, suggesting the importance of basophils for acquired tick resistance (17). However, it remains elusive whether this important finding can be generalized to other animal species including humans. In particular, mice were erroneously believed for a long time to lack basophils, due to the difficulty in identifying them (18-20), and no role in acquired tick resistance in mice has been shown for basophils. Instead, one research group suggests that mast cells in place of basophils are important for acquired immunity to ticks in mice, based on the observation that 
A
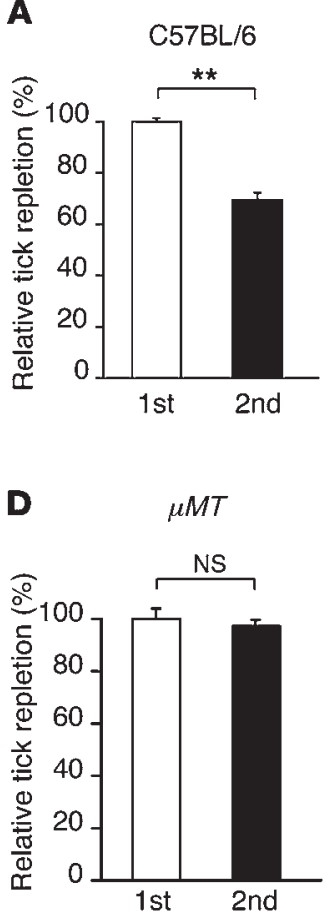

B
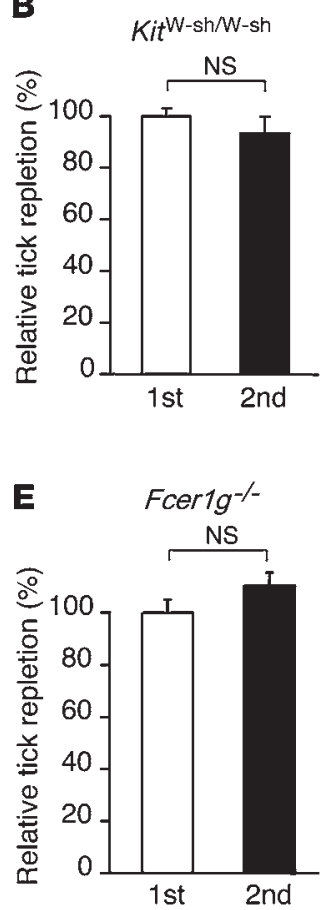
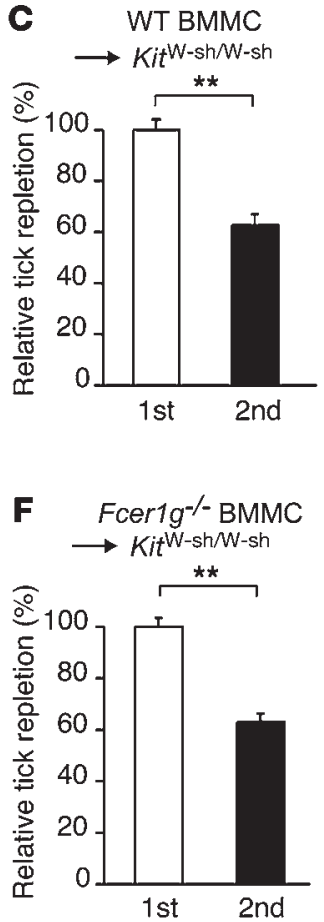

Figure 1

IgFc receptors on mast cells are dispensable for antibody-mediated, protective immunity against ticks. (A) C57BL/6 and (B) C57BL/6-KitW-sh/W-sh mice were infested with larval $H$. longicornis ticks once or twice at an interval of 14 days. The relative tick repletion in each experimental group is shown, in that the value in the first infestation was defined as $100 \%$. Data are shown as mean \pm SEM, $n=5$ (A); $n=4$ (B). (C) BMMCs derived from wild-type mice were adoptively transferred to $K i t^{W-s h} / \mathrm{W}$-sh mice, and the recipient mice were infested and analyzed as in A. Data are shown as mean \pm SEM, $n=3$ each. (D) $\mu M T$ and (E) Fcer1 $g^{-/}$C57BL/6 mice were infested with ticks as in $\mathbf{A}$, and the relative tick repletion in each group is shown. Data are shown as mean \pm SEM, $n=5$ (D); $n=4(E)$. (F) BMMCs derived from Fcer1 $\mathrm{g}^{-/-}$mice were adoptively transferred to $\mathrm{Kit}{ }^{W-s h} / \mathrm{W}$-sh mice, and the recipient mice were infested and analyzed as in A. Data are shown as mean \pm SEM, $n=3$ each. Data shown in $\mathbf{A}-\mathbf{F}$ are representative of at least 3 repeated experiments. ${ }^{* \star} P<0.01$. mast cell-deficient mice failed to acquire tick resistance and that basophils were not detected in tick-feeding sites (21-23). On the other hand, another group showed that the same strain of mast cell-deficient mice developed resistance to another tick species $(24,25)$. Thus, the mechanism underlying acquired immunity to ticks, including the differential roles played by basophils and mast cells, and the influence of genetic background of both ticks and hosts remain to be clarified.

Studies on basophils, particularly their functions in vivo, have long been hampered by the lack of suitable animal models, including mice that are deficient only in basophils. We have recently established a CD200R3-specific mAb Ba103 $(26,27)$ that can deplete most basophils when administered into mice. MAR-1, a mAb specific to FceRI $\alpha$, also shows a similar basophil-depleting ability (28, 29). The use of these mAbs successfully identified previously unrecognized roles for basophils in allergic reactions and the regulation of acquired immunity (30-33), including IgE-mediated chronic cutaneous allergic inflammation $(26,34)$, IgG-mediated systemic anaphylaxis (35), and promotion of humoral memory responses (29) and Th2 cell differentiation $(28,36-38)$. Thus, the mAbs are very useful for analyses of in vivo functions of basophils. However, they might not be perfect in some experimental settings, especially those in which mast cells substantially contribute to a reaction of interest, since the $\mathrm{mAbs}$ react to both mast cells and basophils (26, $27,29)$. Possible side effects of the treatment with the mAbs have to be considered, including activation, unresponsiveness after activation, or partial depletion of mast cells (29). This may make the interpretation of obtained data complicated, whether the phenotype of antibody-treated mice is due to either the basophil depletion or some deleterious effects on mast cells or both.

In the present study, to overcome the limitation of antibodymediated basophil depletion, we utilized the technology of diphtheria toxin-mediated (DT-mediated) cell ablation (39) and generated engineered mice that allow selective ablation of basophils by means of basophil-specific expression of receptors for DT. Taking advantage of this mouse model, we examined the possible involvement of basophils in acquired tick resistance to Haemaphysalis longicornis in mice, for which mast cells are reportedly essential (21-23). H. longicornis is an important vector for human and animal pathogens, including those causing babesiosis, Q fever, and Russian encephalitis (40). We found that basophils but not mast cells directly contribute to the antibody/IgFc receptor-mediated manifestation of tick resistance, even though both types of cells are required for tick resistance.

\section{Results}

IgFc receptors on mast cells are dispensable for antibody-mediated, protective immunity against ticks. Acquired tick resistance is characterized by reduced numbers and weights of engorged ticks in the second infestation compared with the first. Therefore, to evaluate tick resistance, we used "relative tick repletion" (defined in Methods) as a quantitative measure of tick feeding that takes into account both the number and body weight of engorged ticks. The stronger the tick resistance, the lower the relative tick repletion in the second infestation. When C57BL/6 mice were infested with larval H. longicornis ticks twice at an interval of 14 days, the tick repletion in the second infestation typically decreased to approximately $60 \%-70 \%$ of that in the first one (Figure 1A and Supplemental Table 1; supplemental material available online with this article; doi:10.1172/ JCI42680DS1). In contrast, mast cell-deficient C57BL/6-Kit ${ }^{W-s b / W-s h}$ mice showed approximately $100 \%$ tick repletion in the second infestation as in the first one (Figure 1B). Adoptive transfer of bone marrow-derived mast cells (BMMCs) from wild-type $\mathrm{C} 57 \mathrm{BL} / 6$ mice into the skin of C57BL/6-Kit ${ }^{W-s h / W-s h}$ mice resulted in approximately $60 \%$ tick repletion in the second infestation (Figure $1 \mathrm{C}$ ), as observed in wild-type $\mathrm{C} 57 \mathrm{BL} / 6$ mice (Figure $1 \mathrm{~A}$ ). These results demonstrated that $\mathrm{C} 57 \mathrm{BL} / 6$ mice acquire the resistance to tick feeding once they have experienced the tick infestation and 
A

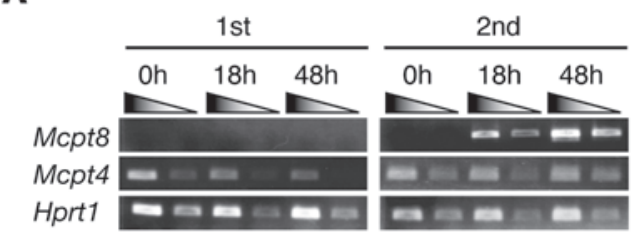

B

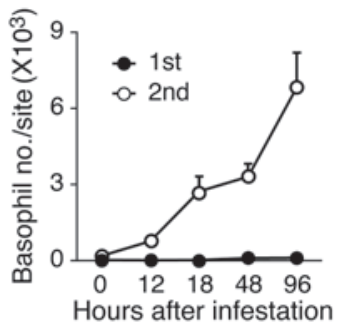

C
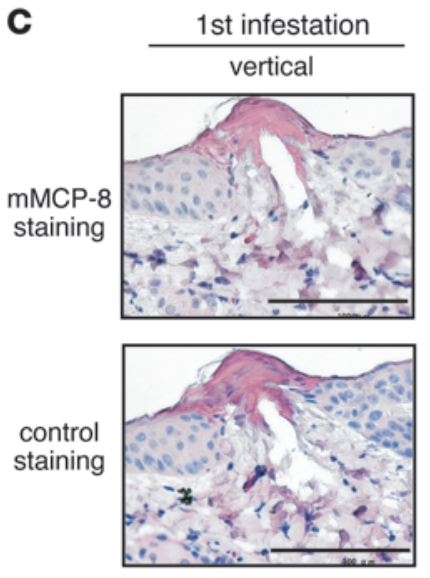

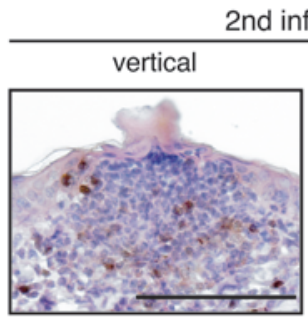

2nd infestation

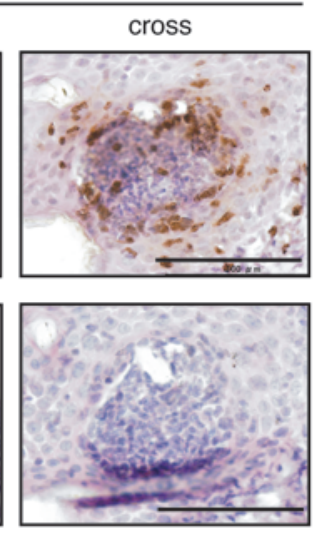

\section{Figure 2}

Recruitment of basophils to tick-feeding sites during the second, but rarely the first, infestation. (A) C57BL/6 mice were infested with ticks as in Figure 1. Tick-feeding-site skin lesions were isolated at the indicated time points during the first and second infestations and subjected to semiquantitative RT-PCR analysis (PCR templates were 5-fold serially diluted) for the expression of basophil-specific Mcpt8, mast cell-specific Mcpt4, and housekeeping Hprt1 transcripts. (B) Skin lesions caused by tick feeding were isolated at the indicated time points during the first (black circles) and second (white circles) infestations and subjected to flow cytometric analysis. Changes in the number of basophils during the infestations are shown. Data are shown as mean \pm SEM, $n=3$ each. (C) Tick-feeding-site skin lesions were isolated 18 hours after the initiation of the first or second infestation and subjected to immunohistochemical examination using anti-mMCP-8 or an isotype-matched control antibody. mMCP-8-expressing basophils were stained in brown. Scale bars: $100 \mu \mathrm{m}$. Data shown in $\mathbf{A}-\mathbf{C}$ are representative of at least 3 repeated experiments. that mast cells play an important role in the acquisition of tick resistance, consistent with previous reports using other strains of mast cell-sufficient and -deficient mice, $\mathrm{WBB} \mathrm{F} 1^{+/+}$and WBB6F1$K i t^{W / W-v}$, respectively $(21,22)$.

Previous studies showed that transfer of serum from tickinfested but not uninfested mice conferred tick resistance on naive mice and that heat inactivation of the serum at $56^{\circ} \mathrm{C}$ for 2 hours abolished this activity, suggesting that antibodies made against ticks, particularly of the IgE class, are involved in the manifestation of tick resistance (23). In accordance with this, neither antibody-deficient $\mu M T$ mice nor Fcer $1 \mathrm{~g}^{-1-}$ mice, which lack the expression of $\operatorname{IgE}$ receptor (FcERI) and stimulatory $\operatorname{IgG}$ receptors, displayed acquired tick resistance in the second infestation (Figure 1, D and E). Unexpectedly, however, mast cell-deficient $K i t^{W-s h / W-s h}$ mice reconstituted with BMMCs derived from Fcer $1 \mathrm{~g}^{-1}$ mice did develop tick resistance (Figure $1 \mathrm{~F}$ ), as did mice reconstituted with wild-type BMMCs (Figure 1C). This finding demonstrated that Ig receptors on mast cells are dispensable for the antibody-mediated acquired tick resistance and suggested a previously unrecognized mechanism for acquisition of tick resistance, distinct from the proposed scenario that tick antigens stimulate skin-resident mast cells through their binding to IgE/FceRI on the cell surface, leading to an immediate-type hypersensitivity reaction against ticks (23). Given that the surface expression of FceRI is restricted to mast cells and basophils in mice, basophils might be involved in antibody-mediated acquired tick resistance.

Recruitment of basophils to tick-feeding sites during the second, but rarely the first, infestation. Giemsa staining of skin tissue sections failed to detect basophils infiltrating the tick-feeding sites (data not shown), consistent with previous reports $(21,25,41)$. Nevertheless, we readily detected $M c p t 8$ transcripts, which encode the basophil-specific, granzyme B-like protease mMCP-8 (42-44) in tick-feeding-induced skin lesions 18 and 48 hours after the initiation of a second infestation; almost none were detected dur- ing the first infestation (Figure 2A). Flow-cytometric analysis of cells isolated from the tick-feeding sites revealed the presence of infiltrating basophils as early as 12 hours after the initiation of the second infestation, but such basophils were rare during the first one (Figure 2B). The number of basophils at the skin lesions increased approximately 40 times from the beginning to the 96 hour time point of the second infestation (Figure 2B). Finally, an immunohistochemical examination using a recently established anti-mMCP-8 mAb (TUG8) (44) demonstrated that mMCP-8expressing basophils were recruited to and clustered around the tick mouth parts during the second, but rarely the first, infestation (Figure 2C). Other types of cells, including eosinophils and neutrophils, also increased their number in the tick-feeding sites during the second infestation compared with the first one (Supplemental Figure 1).

Treatment of mice with basophil-depleting antibodies abolishes acquired tick resistance. We next examined the possible role for basophils infiltrating the tick-feeding sites. The treatment of mice with the basophil-depleting anti-CD200R3 mAb Ba103 $(26,27)$ before the second infestation (Supplemental Figure 2) resulted in a drastically reduced number of infiltrating basophils (Figure $3 \mathrm{~A}$ ) and approximately $100 \%$ tick repletion in the second infestation (Figure 3B), while approximately $60 \%$ tick repletion (the same as in untreated mice) was seen when a control antibody was used (Figure 3B). Similar results were obtained when another basophil-depleting $\mathrm{mAb}$, MAR-1, specific to FceR1 $\alpha(28,29)$, was used instead of Ba103 (Figure $3 \mathrm{C}$ and Supplemental Figure 2). When used to treat mice prior to the first infestation, neither Ba103 nor MAR-1 had a significant effect on the tick repletion in the first infestation (Figure 3D and data not shown). These results supported the idea that basophils critically contribute to protection against ticks during the second infestation. However, the interpretation of these data was not so simple as compared with that in mast cell-independent reactions such as IgE-mediated chronic cutaneous allergic inflammation (26, 
A

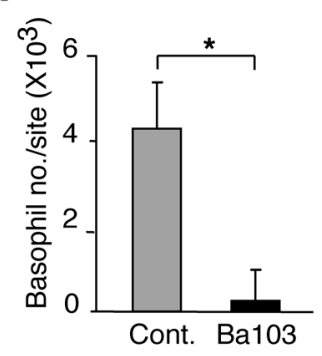

B

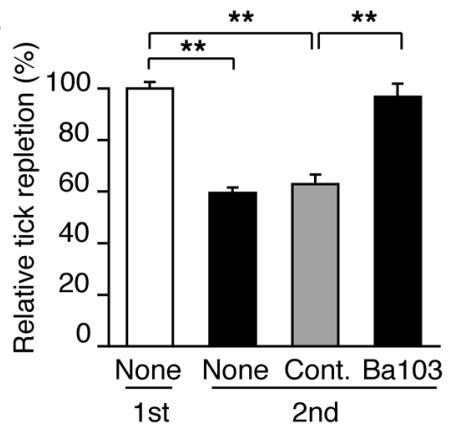

C

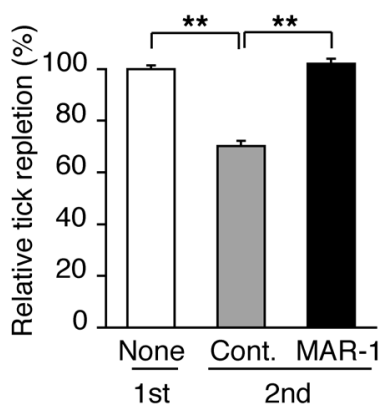

D

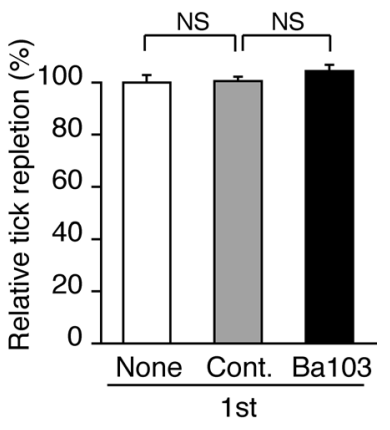

Figure 3

Treatment of mice with basophil-depleting antibodies abolishes acquired tick resistance. (A-C) C57BL/6 mice were infested once or twice and treated with Ba103 (A and B), MAR-1 (C), or an isotype-matched control (Cont) antibody or left untreated (none) before the second infestation. A shows the number of basophils infiltrating the tick-feeding site 2 days after the initiation of the second infestation. Data are shown as mean \pm SEM, $n=3$ each. The relative tick repletion in each experimental group is shown in $\mathbf{B}$ and $\mathbf{C}$, in that the value in the first infestation was defined as $100 \%$. Data are shown as mean \pm SEM, $n=5$ (B); $n=4$ (C). (D) C57BL/6 mice were infested once with ticks after the treatment of Ba103 or control antibody or without any treatment (none). Data are shown as mean \pm SEM, $n=4$ each. Data shown in A-D are representative of at least 3 repeated experiments. ${ }^{*} P<0.01 ;{ }^{*} P<0.05$.

34), since both Ba103 and MAR-1 react with mast cells as well as basophils $(26,27,29)$. Mast cell-deficient mice failed to acquire tick resistance (Figure 1B), and therefore we could not formally exclude the possibility that the treatment of mice with Ba103 or MAR-1 had a deleterious effect on mast cell functions that led to the loss of tick resistance.

Generation of $M c p t 8^{D T R}$ mice for selective and inducible ablation of basophils. To overcome the limitation of antibody-mediated basophil depletion in discrimination of in vivo functions between basophils and mast cells, we generated $M c p t 8^{D T R}$ mice that express the human DT receptor (DTR) only in basophils, which makes it possible to specifically and inducibly deplete basophils by treatment with DT. These mice harbor a cDNA-encoding DTR fused to GFP and an internal ribosome entry site (IRES) inserted into the $3^{\prime}$ untranslated region of the basophil-specific Mcpt8 gene (Figure 4A). As expected, GFP was expressed only in the basophils of Mcpt8DTR mice and not in other cell types, including mast cells, neutrophils, or eosinophils (Figure 4B). DT injection into the $M c p t{ }^{D T R}$ mice led to the transient depletion of basophils from the bone marrow, peripheral blood, and spleen (Figure 4, C and D, and Supplemental Figure 3) but did not affect other cell types, including peritoneal and dermal mast cells (Figure 4, C and E, and Supplemental Figure 3). IgE-mediated, passive cutaneous anaphylaxis was elicited normally in the DT-treated $M c p t{ }^{D T R}$ mice (Figure 4F), demonstrating that the function of the mast cells was also intact. However, tick resistance was abolished when the $M c p t 8^{D T R}$ mice were treated with DT but not vehicle (PBS) before the second infestation (Figure 5A), concomitant with the depletion of basophils, but not mast cells, from the tick-feeding sites (Figure 5, B and C). The DT treatment showed no significant effect on the number of basophils or tick resistance in control littermates (Supplemental Figure 4), and the adoptive transfer of basophils from control littermates reconstituted tick resistance in the DT-treated $M c p t{ }^{D T R}$ mice (Supplemental Figure 5). These findings clearly demonstrated the importance of basophils in the manifestation of tick resistance.

IgFc receptors on basophils are essential for antibody-mediated, protective immunity against ticks. We next examined the possible contribution of basophils to the antibody dependency of acquired tick resistance. The adoptive transfer of basophil-enriched $\mathrm{CD}_{4} 4 \mathrm{~b}^{+}$spleen cells from wild-type mice that had been infested with ticks once, but not of those from uninfested mice, conferred tick resistance on naive mice (Figure 6A). This was also the case when basophilenriched $\mathrm{CD}_{4} \mathrm{~b}^{+}$spleen cells from infested $M c p t 8^{D T R}$ mice were used as the donor cells, and DT-mediated basophil depletion of the donor mice before the transfer abolished the acquisition of tick resistance in the recipients (Figure 6B). These results strongly suggested that anti-tick antibody-armed basophils played a critical role in antibody-mediated tick resistance. Indeed, the adoptive transfer of wild-type, but not Fcer $1 g^{-1}$ basophil-enriched, spleen cells from infested mice conferred tick resistance on naive mice (Figure 6C). Taken together with the result shown in Figure 1F, the expression of Ig receptors on basophils but not mast cells is required for the manifestation of tick resistance.

\section{Discussion}

Basophils account for less than $1 \%$ of peripheral blood leukocytes and share several characteristics with tissue-resident mast cells, including the surface expression of FceRI. Therefore, basophils have long been considered to have minor, redundant roles in immune responses (45). Recent studies have illuminated previously unrecognized roles for basophils in both allergic responses and immune regulation (30-33). However, our understanding of the role of basophils in protective immunity to pathogens is still very limited compared with that of mast cells $(46,47)$. In the present study, we created a mouse strain suitable for analysis of basophil functions in vivo and uncovered the critical role for basophils in acquired resistance against blood-feeding tick H. longicornis in mice. Importantly, basophils but not mast cells are responsible for the antibody/IgFcmediated acquisition of tick resistance, even though both types of cells are essential for the manifestation of resistance.

$M c p t 8^{D T R}$, a mouse model for selective and inducible ablation of basophils established in the present study, is exactly what many researchers studying basophils and mast cells have long been eager for (30-33). The discovery of mast cell-deficient mice, including $K i t^{W / W-v}$ and $K i t^{W-s h / W-s h}$ mice, has greatly advanced our understanding of mast cell functions in vivo (48). In contrast, neither natural nor genetically engineered mutant mice deficient only in basophils were available, which was a great obstacle in the basophil research. 
A

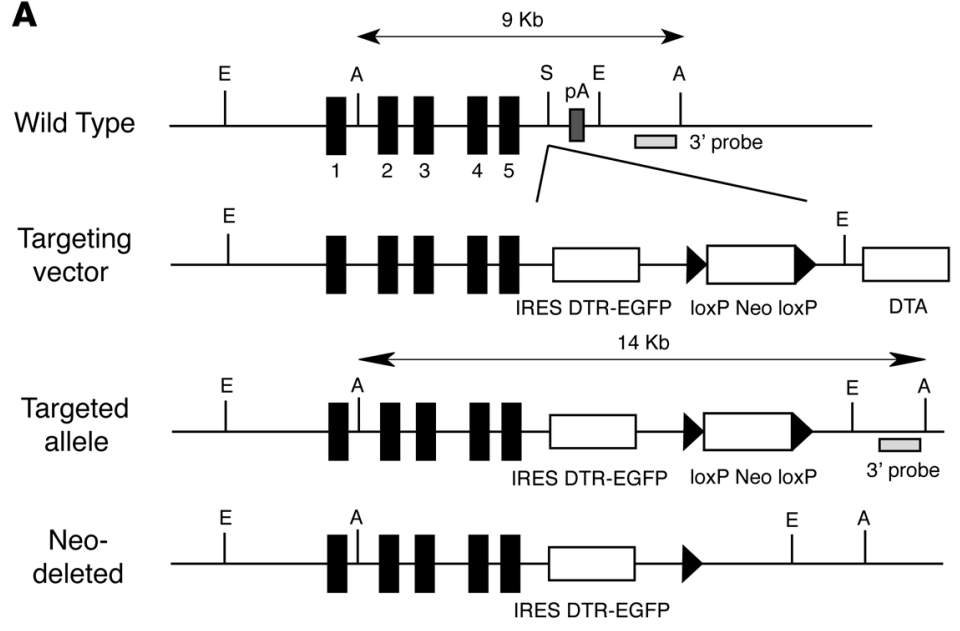

B

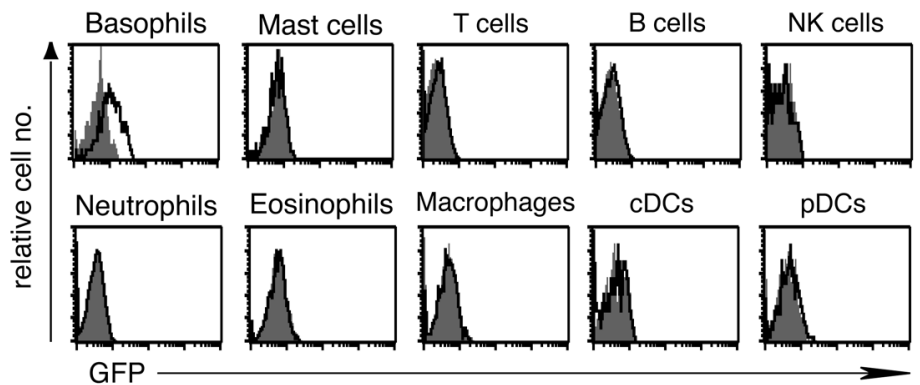

C

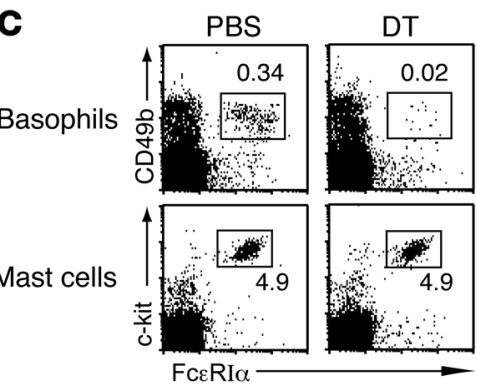

E

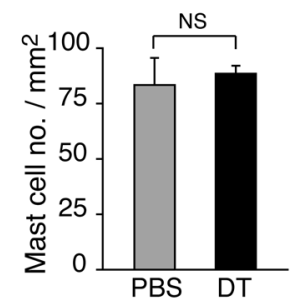

D
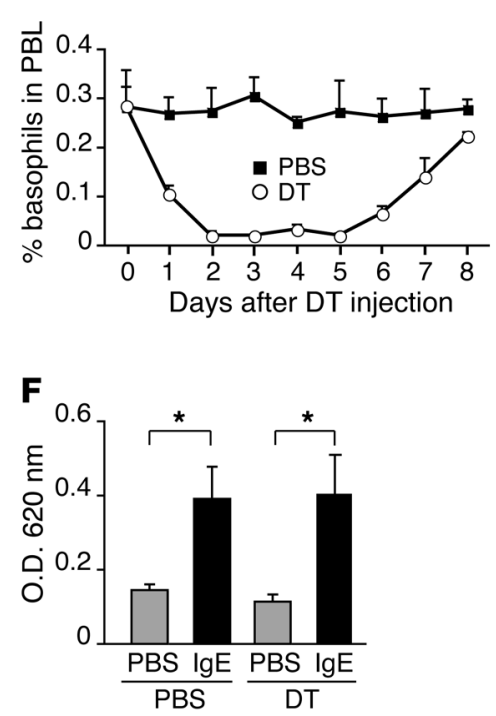

Figure 4

Generation of $M c p t 8^{D T R}$ mice for selective and inducible ablation of basophils. (A) Schematic diagram of Mcpt8DTR targeting construct. Exons are shown as black boxes. The restriction sites indicated are as follows: $\mathrm{E}, E c o R \mathrm{~V}$; S, Sall; A, AfllI. A Sall site engineered in the 3' untranslated region of the Mcpt8 gene was used to clone the IRES-DTR-EGFP-loxP-Neo-loxP cassettes. Neo cassette was removed by Cre-mediated recombination after mating with CAG-cre deleter mice. (B) Flow cytometric analysis of GFP expression in immune cells from Mcpt8 ${ }^{\text {DTR }}$ (white histogram) or wild-type littermate (shaded histogram) mice. Data are shown for basophils, neutrophils, and eosinophils in the bone marrow; mast cells in the peritoneal cavity; and T cells, B cells, NK cells, macrophages, plasmacytoid DCs (pDCs), and conventional DCs (cDCs) in the spleen. (C-F) Mcpt8DTR mice were treated with DT ( $750 \mathrm{ng} / 20 \mathrm{~g}$ body weight) or vehicle (PBS). The percentage of basophils (as indicated by the numbers in the panels) in the bone marrow and mast cells in the peritoneum 3 days after the injection are shown in $\mathbf{C}$. The change in percentage of basophils in the peripheral blood after the injection is plotted in $\mathbf{D}$. The number of mast cells in the flank skin 3 days after the injection is shown in E. Data are shown as mean \pm SEM, $n=3$ each. $(\mathbf{F})$ Mice were subjected to passive cutaneous anaphylaxis 3 days after the DT injection. Data are shown as mean \pm SEM, $n=4$ each. Data shown in $\mathbf{B}-\mathbf{F}$ are representative of at least 3 repeated experiments. ${ }^{*} P<0.05$.

that are permanently deficient for basophils. Taking advantage of the selective and inducible ablation of basophils in $M c p t 8^{D T R}$ mice, we definitely demonstrated that basophils play an essential role in the acquired protective immunity to $H$. longicornis in mice in contrast to the previously proposed scenario that mast cells substitute for basophils in the acquisition of tick resistance in mice, unlike in guinea pigs (23).

Previous studies showed that the number of mast cells increased at tick-feeding sites in mice with acquired tick resistance, whereas no infiltrating basophils were detected $(21,25,41)$. In the present study, by using the recently established anti-mMCP-8 mAb TUG8, which is suitable for immunohistochemical staining of basophils in tissue sections (44), we clearly identified infiltrating basophils that made clusters surrounding tick mouth parts at $H$. longicornis feeding sites during the second but not first infestation. Thus, the absence of infiltrating basophils at the tick-feeding sites as reported in the previous studies $(21,25,41)$ is prob-

The establishment of $M c p t 8^{D T R}$ mice has clearly solved the problem associated with antibody-mediated basophil depletion using Ba103 or MAR-1, which reacts to mast cells as well as basophils $(26,27,29)$, and hence has potential to produce unwanted side effects on mast cells. The expression of DTR in Mcpt8DTR mice is under the control of the $M c p t 8$ promoter and regulatory elements, and the DT treatment depletes only basophils and leaves mast cells intact. Inducible depletion of basophils could also minimize possible compensation of basophil functions by other types of cells, compared with mice ably due to the difficulty in identifying mouse basophils by conventional methods such as Giemsa staining $(19,20)$. We further demonstrated that the selective depletion of basophils abolished the acquired tick resistance. On the other hand, we confirmed the previous observation that the absence of mast cells resulted in the loss of acquired tick resistance (21-23). Thus, both basophils and mast cells critically contribute to the manifestation of tick resistance. However, their contribution does not seem additive, since the absence of either basophils or mast cells led to the com- 
A

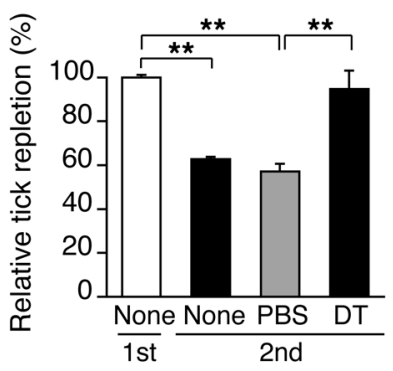

Figure 5

DT-mediated basophil ablation in Mcpt8 ${ }^{\text {DTR }}$ mice abolishes acquired tick resistance. Mcpt8 ${ }^{\text {DTR }}$ mice were infested once or twice with ticks as in Figure 1. DT or vehicle (PBS) was administered twice, 2 days before and 2 days after the initiation of the second infestation. The relative tick repletion for each group is shown in A. Data are shown as mean $\pm \mathrm{SEM}, n=3$ each. (B and $\mathbf{C}$ ) Numbers of basophils and mast cells in the tick-feeding sites 4 days after the initiation of the second infestation. Data are shown as mean \pm SEM, $n=3$ each. Data shown in $\mathbf{A}-\mathbf{C}$ are representative of at least 3 experiments. ${ }^{* *} P<0.01 ;{ }^{*} P<0.05$.

plete loss of acquired tick resistance, and the antibody-mediated depletion of basophils in mast cell-deficient mice showed no significant effect on tick repletion in the second infestation (data not shown). These results suggested that basophils and mast cells might have distinct roles in the acquisition of tick resistance. Indeed, the expression of $\mathrm{IgFc}$ receptors on basophils but not mast cells was required for it, indicating that the antibody-mediated acquisition of anti-tick immunity is ascribed to basophils rather than mast cells.

It remains to be determined how basophils and mast cells accomplish tick resistance in a cooperative or independent manner. Basophil infiltration in the tick-feeding sites was normally observed even in $K i t^{W-s h / W-s h}$ or $\mu M T$ mice (Supplemental Figure 6 , A and B), demonstrating that neither mast cells nor antibodies are essential for the recruitment of basophils, although both are required for the manifestation of acquired tick resistance. Moreover, the recruitment of eosinophils and neutrophils was animal species, including guinea pigs. Thus, the extent of contribution of mast cells to acquired tick resistance may depend on the combination of host animal and tick species. Given that basophils cluster closer to tick mouth parts than mast cells, we assume that basophils rather than mast cells function as direct effectors of the anti-tick reaction mounted in response to tick antigens in our mouse model. The effector molecules involved in the manifestation of tick resistance remain to be identified. Immunohistochemical staining for $\mathrm{mMCP}-8$ indicated that the basophils that were adjacent to tick mouth parts had degranulated to release mMCP-8 (Figure 2C), which is a basophil-specific, granzyme B-like serine protease that is stored in the secretory granules of basophils (42-44). We recently reported that a tryptase, mMCP-11, is also preferentially expressed by basophils and stored in their secretory granules (44). The mast cell protease mMCP- 6 has been shown to play an immunoprotective role in bacterial and helminth infections $(49,50)$. Therefore, the
A

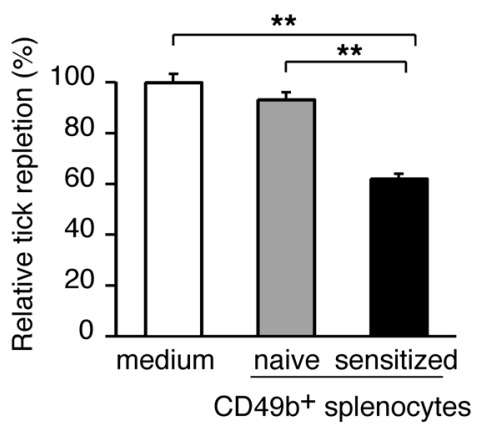

B

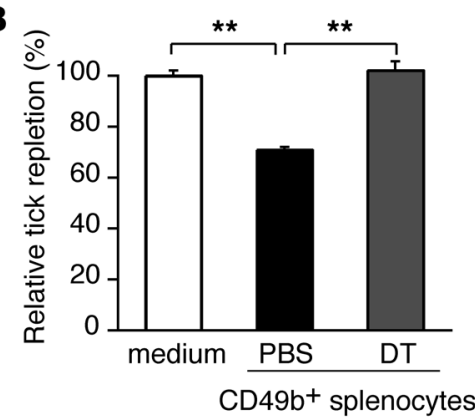

C

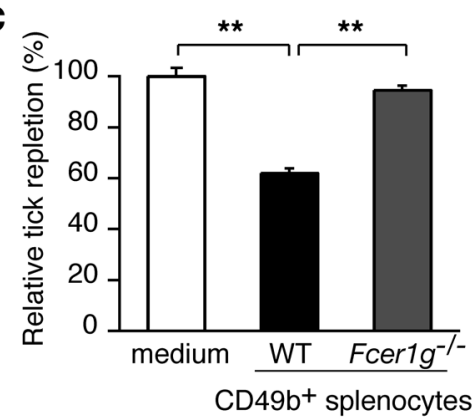

Figure 6

IgFc receptors on basophils are essential for antibody-mediated, protective immunity against ticks. (A) The CD49b+ basophil-enriched fraction of splenocytes from C57BL/6 mice infested only once (sensitized) or never (naive) was adoptively transferred into naive, uninfested C57BL/6 mice, and 2 hours later, the recipient mice were infested with ticks. The relative tick repletion for each group is shown. The value for the control, vehicle-treated mice was defined as $100 \%$. Data are shown as mean \pm SEM, $n=3$ each. (B) The basophil-enriched fraction of splenocytes from DT- or PBS-treated Mcpt8DTR mice that had been infested once was adoptively transferred into naive, uninfested littermate control mice. The recipient mice were then infested, and the relative tick repletion is shown as in $\mathbf{A}$. Data are shown as mean $\pm \mathrm{SEM}, n=3$ each. (C) The basophilenriched fraction of splenocytes from wild-type or Fcer $1 \mathrm{~g}^{-1-}$ mice that had been infested once was adoptively transferred into naive, uninfested $\mathrm{C} 57 \mathrm{BL} / 6$ mice, which were then infested. The relative tick repletion is shown as in $\mathbf{A}$. Data are shown as mean $\pm \mathrm{SEM}, n=3$ each. Data shown in $\mathbf{A}-\mathbf{C}$ are representative of at least 3 repeated experiments. ${ }^{* \star} P<0.01$. 
basophil-derived mMCP-8 and mMCP-11, among other factors, may contribute to tick resistance. Further studies, including the generation of mice deficient for these proteases, are needed to explore this possibility.

Recent studies demonstrated that basophils can enter lymph nodes, initiate Th2 responses, and enhance humoral memory responses in certain experimental settings $(28,29,36-38)$. The infestation of mice with $H$. longicornis greatly increases serum IgE levels (23), suggesting that the tick infestation induces Th2-type immune responses. We detected the recruitment of basophils expressing MHC class II and CD86 to regional lymph nodes during the first infestation with ticks (Supplemental Figure $8 \mathrm{~A})$. However, the DT-mediated basophil depletion in the first infestation did not show any significant effect on increased levels of serum IgE (Supplemental Figure $8 \mathrm{~B}$ ) or the manifestation of acquired tick resistance in the second infestation (Supplemental Figure 9), although it abolished the basophil recruitment to the lymph nodes (Supplemental Figure 8A). The basophil depletion in the second infestation also showed no significant impact on serum IgE levels (Supplemental Figure 8B), even though it abolished acquired tick resistance (Figure 5A). These results imply that the loss of acquired tick resistance in the basophil-depleted mice may not be due to the failure in Th2-induced IgE production. Moreover, as shown in Figure 6, the adoptive transfer of basophils from infested mice conferred tick resistance on naive mice in that $\mathrm{T}$ cells and $\mathrm{B}$ cells had not been primed with tick antigens. This suggests that the enhancement of $\mathrm{T}$ and $\mathrm{B}$ cell memory responses mediated by basophils, if any, may not be essential for the manifestation of acquired tick resistance, even though our observations do not exclude roles for basophils in the promotion of Th2 response and the enhancement of memory response during tick infestation.

The present study reconciles the apparent discrepancy between guinea pigs and mice in the cellular components each requires for acquired tick resistance $(17,21-25)$ and illuminates a nonredundant role for basophils in protective immunity to ectoparasites. The acquisition of tick resistance is associated with reduced pathogen transmission from infected ticks (12-15), and therefore our findings may provide new avenues toward the development of novel control strategies for tick infestations and tick-borne diseases. Finally, since the study of basophils has long been hampered by the lack of basophil-deficient animal models, the establishment of $M c p t 8^{D T R}$ mice in the present study should facilitate our understanding of the in vivo roles played by basophils under physiological and pathological conditions.

\section{Methods}

Mice. C57BL/6 mice were purchased from Japan SLC. Fcer $1 g^{-1}, \mu M T$, and $K i t^{W-s h / W-s h}$ C57BL/6 mice were described previously (51-53). Kit ${ }^{W-s h / W-s h}$ C57BL/6 mice were provided by S.J. Galli (Stanford University, Stanford, California, USA), S. Nakae (University of Tokyo, Tokyo, Japan), and K. Sudo (Tokyo Medical University, Tokyo, Japan). CAG-cre transgenic mice were provided by J. Miyazaki (Osaka University, Osaka, Japan). Mice were maintained under specific pathogen-free conditions in our animal facilities. All animal studies were approved by the Animal Research Committee of Tokyo Medical and Dental University and the Animal Care Committee of the Jikei University School of Medicine.

Generation of $M c p t 8^{D T R}$ mice. The targeting construct used to generate the $M c p t 8^{D T R}$ mice was made by subcloning a $10-\mathrm{kbp} E c o R V$ fragment of $M c p t 8$ containing exons 1-5 from a C57BL/6 mouse genomic DNA BAC library
(BPRC) into the pBluescript II (Stratagene) vector. During this step, the SalI site was destroyed, and a PMC1-DTA negative-selection cassette was then inserted into the SmaI site. A SalI restriction site was engineered into the $3^{\prime}$ untranslated region of $M c p t 8$, upstream of the polyadenylation signal. The PCR-amplified IRES-huDTR cDNA as an EcoRI/BamHI fragment was cloned into PEGFP-N3 (Stratagene) to generate the gene encoding the IRES-huDTR-EGFP fusion protein, which was then cloned into the MCSBGHpA-loxP-Neo-loxP shuttle vector. The IRES-huDTR-EGFP-BGHpAloxP-Neo-loxP fragment was cloned into the above-mentioned SalI site. The linearized targeting vector was electroporated into embryonic stem cells. G418-resistant clones were screened for homologous recombination by Southern blotting analysis. Clones carrying the correctly targeted locus were injected into BDF2 blastocysts to generate chimeric mice. These chimeras were subsequently crossed with C57BL/6 females. Chimeric offspring were mated to CAG-cre transgenic mice (54) to excise the PGK-Neo cassette. Mice were backcrossed to C57BL/ 6 at least 6 times before use.

Ticks and tick infestation. H. longicornis of the Okayama strain was provided by K. Fujisaki (Kagoshima University, Kagoshima, Japan). H. longicornis larvae were used at $4-10$ weeks of age for the cutaneous infestation of mice as described (21). A patch of the mouse's flank was shaved, and a short piece of acrylic pipe with 40 larvae in it was attached to the skin. The open end of the pipe was covered with nylon gauze to prevent the ticks from escaping. Of the 40 larvae applied initially, those that detached over a period lasting up to 8 days were defined as being engorged. Under our experimental conditions, approximately $85 \%$ of the ticks became engorged during the first infestation, regardless of the mouse strain examined, and the rest of them failed to feed and died. The mean body weight of the engorged ticks in the first infestation was typically approximately $750 \mu \mathrm{g}$, regardless of the mouse strain, whereas that of unengorged ticks was only approximately $1-2 \mu \mathrm{g}$. To assess the acquisition of tick resistance, mice were infested with tick larvae at 2 different locations. The initial infestation was on the left flank. Two weeks later, the second infestation was started on the right flank. In mice that had become resistant to ticks, both the number and body weight of the engorged ticks was reduced compared with naive mice (Supplemental Table 1). For evaluation of tick resistance, we summed up the body weight of all engorged ticks in each mouse as our measure of tick feeding and calculated "relative tick repletion." Relative tick repletion (\%) = $100 \times$ the sum of the body weights of all engorged ticks in the test experiment/the sum of the body weights of all engorged ticks in the reference experiment. When the tick repletion in the first infestation was defined as $100 \%$, that in the second infestation was typically approximately $60 \%-70 \%$ in C57BL/ 6 mice. Because the body weight of unengorged ticks is negligible compared with that of engorged ones, the relative tick repletion in the second infestation compared with the first one actually represents the ratio of the total weight of all ticks in the second infestation to that in the first infestation.

Antibodies. PE-conjugated mAbs specific to Siglec-F (E50-2440), c-kit (2B8), NK1.1 (PK136), CD11b (M1/70), CD19 (1D3), and B220 (RA3-6B2) and biotinylated mAbs specific to mouse IgE (R35-72), CD49b (DX5), CD11c (HL3), c-kit (2B8), CD40 (3/23), and CD86 (GL-1) were purchased from BD Biosciences - Pharmingen. Unlabeled and PE-conjugated antimouse FceRI $\alpha$ (MAR-1) and biotinylated anti-Gr-1 (RB6-8C5), anti-F4/80 (BM8), anti-CD3 (145-2C11), MHC class II (M5/114.15.2), and CD80 (16-10A1) were from eBioscience. The HRP-conjugated goat anti-rat IgG was obtained from Santa Cruz Biotechnology Inc. The basophil-depleting, anti-CD200R3 $\mathrm{mAb}$ (Ba103) and the anti-mMCP-8 mAb (TUG8) were established as reported previously $(26,27,44)$.

Semiquantitative RT-PCR analysis. Total RNA was prepared from pieces of skin taken from tick-feeding sites and subjected to first-strand cDNA synthesis with reverse transcription using oligo(dT)primers. Semiquantitative PCR was performed with 5-fold serially diluted cDNA templates using the following prim- 
ers: for $M c p t 8$, forward, 5'-CCGGAATTCATGTTCCTGCTCCTGGTCC-3' and reverse 5'-CGCGGATCCCTAGGGTTGTTGCAGGAGTTTCATTG-3'; for $M c p t 4$, forward $5^{\prime}$-GGGCTGGAGCTGAGGAGATT-3' and reverse 5'-CTTCTGCTGTGTGGATTCTGTC-3'; and for Hprt1, forward, 5'-GCTGGTGAAAAGGACCTCT-3' and reverse 5'-CACAGGACTAGAACACCTGC-3'.

Flow cytometric and histological analyses. Pieces of skin from the tick-feeding sites were treated with collagenase ( $130 \mathrm{U} / \mathrm{ml}$, Wako Pure Chemical) in RPMI complete medium at $37^{\circ} \mathrm{C}$ for 2 hours to obtain single-cell suspensions. Cells prepared from skin, bone marrow, the peritoneal cavity, and peripheral blood were depleted of rbcs by lysis with hypotonic buffer and preincubated with anti-CD16/32 (2.4G2) mAb (BD Biosciences - Pharmingen) and normal rat serum on ice for 10 minutes to prevent the nonspecific binding of other Abs. Cells were stained with the indicated combinations of Abs and analyzed by FACSCalibur (BD Biosciences): basophils (IgE ${ }^{+} \mathrm{c}-\mathrm{kit}^{-}$or FceRI $\alpha^{+} \mathrm{CD} 49 \mathrm{~b}^{+}$), mast cells $\left(\mathrm{IgE}^{+} \mathrm{c}-\mathrm{kit}^{+}\right.$or FceRI $\left.\alpha^{+} \mathrm{c}-\mathrm{kit}^{+}\right)$, eosinophils $\left(\mathrm{Gr}-1^{\text {int }}\right.$ siglec- $\left.\mathrm{F}^{+}\right)$, neutrophils $\left(\mathrm{Gr}-1^{\text {hi }}\right), \mathrm{T}$ cells $\left(\mathrm{CD}^{+}\right)$, B cells $\left(\mathrm{CD} 19^{+}\right)$, NK cells $\left(\mathrm{NK} 1.1^{+} \mathrm{CD} 49 \mathrm{~b}^{+}\right)$, monocytes/macrophages (Gr- $1^{\text {int }}$ Siglec- $\left.\mathrm{F}^{-}\right)$, macrophages $\left(\mathrm{CD} 11 \mathrm{~b}^{+} \mathrm{F} 4 / 80^{+}\right)$, plasmacytoid DCs (pDCs, CD11 $\left.\mathrm{c}^{\text {int }} \mathrm{B} 220^{+}\right)$, and conventional DCs $(\mathrm{cDCs}$, CD11 $\left.{ }^{\text {hiB }}{ }^{2} 20^{-}\right)$. For immunohistochemical examination, paraffin-embedded skin specimens were prepared and pretreated as described (44), and tissue sections were incubated with anti-mMCP-8 $(1 \mu \mathrm{g} / \mathrm{ml})$ or an isotype-matched control antibody at $4{ }^{\circ} \mathrm{C}$ overnight, followed by HRP-conjugated goat antirat $\operatorname{IgG}$ (diluted 1:500). The sections were subsequently incubated in a DAB solution (Dako) and counterstained with H\&E. Mast cells in the skin tissue sections were identified by toluidine blue staining ( $\mathrm{pH} 2.5$ ).

Basophil depletion. For the antibody-mediated depletion of basophils, mice were treated with either Ba103 or MAR-1. One intravenous injection of $50 \mu \mathrm{g}$ $\mathrm{Ba} 103$ or control rat IgG was given 1 day before the tick infestation. A total of $30 \mu \mathrm{g}$ MAR-1 or control hamster IgG was administered intraperitoneally twice a day ( $5 \mu \mathrm{g}$ each time) for 3 consecutive days, starting 4 days before the tick infestation. For the DT-mediated depletion, $M c p t 8^{D T R}$ mice were given an intravenous injection of DT (750 ng/20 g body weight; Sigma-Aldrich).

Adoptive transfer of cells. Mouse bone marrow cells were cultured with $3.5 \mathrm{ng} / \mathrm{ml}$ rIL-3 and $15 \mathrm{ng} / \mathrm{ml} \mathrm{rSCF}$ for approximately 4-6 weeks to obtain BMMCs. A total of $10^{6} \mathrm{BMMCs}$ was injected in 10 separate intradermal injections to form a circle (16 $\mathrm{mm}$ in diameter) on the right flank of $K i t^{W-s h / W-s h}$ mice. Four weeks later, the mice were infested with tick larvae, first on the left flank and then, 2 weeks later, by a second infestation in which the larvae were placed within the BMMC-injected site. For the adoptive transfer of basophils, spleen cells were isolated from mice that were uninfested or infested with ticks 2 weeks previously, and the basophil-enriched $\mathrm{CD} 49 \mathrm{~b}^{+}$fraction was prepared by using the BD IMag Cell Separation System (BD Biosciences - Pharmingen). Mice were infested with ticks 2 hours after the adoptive transfer of the $\mathrm{CD}_{49 \mathrm{~b}^{+}}$fraction $\left(1.5 \times 10^{6}\right.$ cells $)$.

Passive cutaneous anaphylaxis. Mice were passively sensitized with an intradermal injection of TNP-specific IgE or control PBS into the ear and challenged 24 hours later with an intravenous injection of TNP-OVA plus $0.5 \%$ Evans blue dye as described. The absorbance of Evans blue in the ear extract was measured with a spectrophotometer at $620 \mathrm{~nm}$.

Statistics. Statistical significance $(P<0.05)$ was determined by 2 -tailed Student's $t$ test or ANOVA followed by Ryan's test.

\section{Acknowledgments}

We thank K. Fujisaki for providing ticks; S.J. Galli, S. Nakae, and K. Sudo for providing Kit ${ }^{W-s h / W-s h}$ C57BL/ 6 mice; J. Miyazaki for providing CAG-cre transgenic mice; $\mathrm{M}$. Matsuda for helping with the generation of $M c p t 8^{D T R}$ mice; and M. Egawa and H. Ogawa for breeding mice. This work was supported by research grants from JST, CREST, the Japanese Ministry of Education, Culture, Sports, Science and Technology, Takeda Science Foundation, the Mitsubishi Foundation, the Naito Foundation, and the Uehara Memorial Foundation.

Received for publication February 16, 2010, and accepted in revised form May 19, 2010.

Address correspondence to: Hajime Karasuyama, Department of Immune Regulation, Tokyo Medical and Dental University Graduate School, 1-5-45 Yushima, Bunkyo-ku, Tokyo 113-8519, Japan. Phone: 81.3.5803.5162; Fax: 81.3.3814.7172; E-mail: karasuyama. mbch@tmd.ac.jp.
1. Parola P, Raoult D. Ticks and tickborne bacterial diseases in humans: an emerging infectious threat. Clin Infect Dis. 2001;32(6):897-928.

2. de la Fuente J, Estrada-Pena A, Venzal JM, Kocan $\mathrm{KM}$, Sonenshine DE. Overview: Ticks as vectors of pathogens that cause disease in humans and animals. Front Biosci. 2008;13:6938-6946.

3. Wikel SK. Tick modulation of host immunity: an important factor in pathogen transmission. Int J Parasitol. 1999;29(6):851-859.

4. Nuttall PA, Paesen GC, Lawrie CH, Wang H. Vector-host interactions in disease transmission. $\mathrm{J} \mathrm{Mol}$ Microbiol Biotechnol. 2000;2(4):381-386.

5. Kovar L. Tick saliva in anti-tick immunity and pathogen transmission. Folia Microbiol (Praba). 2004;49(3):327-336.

6. Wikel SK. Host immunity to ticks. Annu Rev Entomol. 1996;41:1-22.

7. Willadsen P, Jongejan F. Immunology of the tickhost interaction and the control of ticks and tickborne diseases. Parasitol Today. 1999;15(7):258-262.

8. Brossard M, Wikel SK. Tick immunobiology. Parasitology. 2004;129(suppl):S161-S176.

9. Trager W. Acquired immunity to ticks. J Parasitol. 1939;25:57-81.

10. Allen JR. Tick resistance: basophils in skin reactions of resistant guinea pigs. Int J Parasitol. 1973;3(2):195-200.

11. Brown SJ, Askenase PW. Cutaneous basophil responses and immune resistance of guinea pigs to ticks: passive transfer with peritoneal exudate cells or serum. J Immunol. 1981;127(5):2163-2167.
12. Francis J, Little DA. Resistance of droughtmaster cattle to tick infestation and babesiosis. Aust Vet J. 1964;40(7):247-253.

13. Wikel SK. Host resistance to tick-borne pathogens by virtue of resistance to tick infestation. Ann Trop Med Parasitol. 1980;74(1):103-104.

14. Jones LD, Nuttall PA. The effect of host resistance to tick infestation on the transmission of Thogoto virus by ticks. J Gen Virol. 1990;71(pt 5):1039-1043.

15. Wikel SK, Ramachandra RN, Bergman DK, Burkot TR, Piesman J. Infestation with pathogen-free nymphs of the tick Ixodes scapularis induces host resistance to transmission of Borrelia burgdorferi by ticks. Infect Immun. 1997;65(1):335-338.

16. Askenase PW. Immunopathology of parasitic diseases: involvement of basophils and mast cells. Springer Semin Immunopathol. 1980;2(4):417-442.

17. Brown SJ, Galli SJ, Gleich GJ, Askenase PW. Ablation of immunity to Amblyomma americanum by anti-basophil serum: cooperation between basophils and eosinophils in expression of immunity to ectoparasites (ticks) in guinea pigs. J Immunol. 1982;129(2):790-796

18. Urbina C, Ortiz C, Hurtado I. A new look at basophils in mice. Int Arch Allergy Appl Immunol. 1981;66(2):158-160.

19. Dvorak AM, Nabel G, Pyne K, Cantor H, Dvorak HF, Galli SJ. Ultrastructural identification of the mouse basophil. Blood. 1982;59(6):1279-1285.

20. Dvorak AM. The mouse basophil, a rare and rarely recognized granulocyte. Blood. 2000;96(4):1616-1617.

21. Matsuda H, Fukui K, Kiso Y, Kitamura Y. Inabil- ity of genetically mast cell-deficient W/Wv mice to acquire resistance against larval Haemaphysalis longicornis ticks. J Parasitol. 1985;71(4):443-448.

22. Matsuda H, Nakano T, Kiso Y, Kitamura Y. Normalization of anti-tick response of mast cell-deficient $\mathrm{W} / \mathrm{Wv}$ mice by intracutaneous injection of cultured mast cells. J Parasitol. 1987;73(1):155-160.

23. Matsuda $H$, et al. Necessity of IgE antibodies and mast cells for manifestation of resistance against larval Haemaphysalis longicornis ticks in mice. J Immunol. 1990;144(1):259-262.

24. denHollander N, Allen JR. Dermacentor variabilis: resistance to ticks acquired by mast celldeficient and other strains of mice. Exp Parasitol. 1985;59(2):169-179.

25. Steeves EB, Allen JR. Tick resistance in mast celldeficient mice: histological studies. Int J Parasitol. 1991;21(2):265-268.

26. Obata K, et al. Basophils are essential initiators of a novel type of chronic allergic inflammation. Blood. 2007;110(3):913-920.

27. Kojima T, et al. Mast cells and basophils are selectively activated in vitro and in vivo through CD200R3 in an IgE-independent manner. J Immunol. 2007;179(10):7093-7100.

28. Sokol CL, Barton GM, Farr AG, Medzhitov R. A mechanism for the initiation of allergeninduced T helper type 2 responses. Nat Immunol. 2008;9(3):310-318.

29. Denzel A, et al. Basophils enhance immunological memory responses. Nat Immunol. 2008;9(7):733-742.

30. Min B. Basophils: what they 'can do' versus what they 
'actually do'. Nat Immunol. 2008;9(12):1333-1339.

31. Karasuyama H, Mukai K, Tsujimura Y, Obata K. Newly discovered roles for basophils: a neglected minority gains new respect. Nat Rev Immunol. 2009;9(1):9-13.

32. Sullivan BM, Locksley RM. Basophils: a nonredundant contributor to host immunity. Immunity. 2009;30(1):12-20.

33. Sokol CL, Medzhitov R. Emerging functions of basophils in protective and allergic immune responses. Mucosal Immunol. 2010;3(2):129-137.

34. Mukai K, et al. Basophils play a critical role in the development of IgE-mediated chronic allergic inflammation independently of T cells and mast cells. Immunity. 2005;23(2):191-202.

35. Tsujimura $Y$, et al. Basophils play a pivotal role in immunoglobulin-G-mediated but not immunoglobulin-E-mediated systemic anaphylaxis. Immunity. 2008;28(4):581-589.

36. Perrigoue JG, et al. MHC class II-dependent basophil-CD4(+) $\mathrm{T}$ cell interactions promote $\mathrm{T}(\mathrm{H}) 2$ cytokine-dependent immunity. Nat Immunol. 2009;10(7):697-705.

37. Sokol CL, Chu NQ, Yu S, Nish SA, Laufer TM, Medzhitov R. Basophils function as antigen-presenting cells for an allergen-induced T helper type 2 response. Nat Immunol. 2009;10(7):713-720.

38. Yoshimoto T, et al. Basophils contribute to $\mathrm{T}(\mathrm{H}) 2$ $\mathrm{IgE}$ responses in vivo via IL-4 production and presentation of peptide-MHC class II complexes to
CD4(+) T cells. Nat Immunol. 2009;10(7):706-712.

39. Saito M, et al. Diphtheria toxin receptor-mediated conditional and targeted cell ablation in transgenic mice. Nat Biotechnol. 2001;19(8):746-750.

40. Tsuji N, Miyoshi T, Battsetseg B, Matsuo T, Xuan $\mathrm{X}$, Fujisaki K. A cysteine protease is critical for Babesia spp. transmission in Haemaphysalis ticks. PLoS Pathog. 2008;4(5):e1000062.

41. denHollander N, Allen JR. Dermacentor variabilis: acquired resistance to ticks in BALB/c mice. Exp Parasitol. 1985;59(1):118-129.

42. Poorafshar M, Helmby H, Troye-Blomberg M, Hellman L. MMCP-8, the first lineage-specific differentiation marker for mouse basophils. Elevated numbers of potent IL-4-producing and MMCP-8positive cells in spleens of malaria-infected mice. Eur J Immunol. 2000;30(9):2660-2668.

43. Lunderius C, Hellman L. Characterization of the gene encoding mouse mast cell protease 8 ( $\mathrm{mMCP}-8$ ), and a comparative analysis of hematopoietic serine protease genes. Immunogenetics. 2001;53(3):225-232.

44. Ugajin $T$, et al. Basophils preferentially express mouse Mast Cell Protease 11 among the mast cell tryptase family in contrast to mast cells. J Leukoc Biol. 2009;86(6):1417-1425.

45. Galli SJ. Mast cells and basophils. Curr Opin Hematol. 2000;7(1):32-39.

46. Falcone FH, Pritchard DI, Gibbs BF. Do basophils play a role in immunity against parasites? Trends Parasitol. 2001;17(3):126-129.
47. Voehringer D. The role of basophils in helminth infection. Trends Parasitol. 2009;25(12):551-556.

48. Galli SJ, Kalesnikoff J, Grimbaldeston MA, Piliponsky AM, Williams CM, Tsai M. Mast cells as "tunable" effector and immunoregulatory cells: recent advances. Annu Rev Immunol. 2005;23:749-786.

49. Thakurdas SM, et al. The mast cell-restricted tryptase mMCP- 6 has a critical immunoprotective role in bacterial infections. $J$ Biol Chem. 2007;282(29):20809-20815.

50. Shin K, et al. Mouse mast cell tryptase mMCP-6 is a critical link between adaptive and innate immunity in the chronic phase of Trichinella spiralis infection. J Immunol. 2008;180(7):4885-4891.

51. Takai T, Li M, Sylvestre D, Clynes R, Ravetch JV. $\mathrm{FcR}$ gamma chain deletion results in pleiotrophic effector cell defects. Cell. 1994;76(3):519-529.

52. Kitamura D, Roes J, Kuhn R, Rajewsky K. A B cell-deficient mouse by targeted disruption of the membrane exon of the immunoglobulin mu chain gene. Nature. 1991;350(6317):423-426.

53. Grimbaldeston MA, Chen CC, Piliponsky AM, Tsai M, Tam SY, Galli SJ. Mast cell-deficient W-sash c-kit mutant Kit W-sh/W-sh mice as a model for investigating mast cell biology in vivo. Am J Pathol. 2005;167(3):835-848.

54. Sakai K, Miyazaki J. A transgenic mouse line that retains Cre recombinase activity in mature oocytes irrespective of the cre transgene transmission. Biochem Biophys Res Commun. 1997;237(2):318-324. 